\title{
Homéostasie du fer et autophagie
}

Ahmed Hamaï ${ }^{1,2}$, Maryam Mehrpour ${ }^{1,2}$

> Le fer est un oligo-élément indispensable à la vie. Cependant, la capacité du fer à alterner entre formes oxydées et réduites contribue à la formation d'espèces réactives de l'oxygène. La génération de ces radicaux libres conduit à un stress oxydatif et à l'initiation des voies de signalisation impliquées dans la survie ou la mort cellulaire. L'homéostasie du fer est donc très soigneusement régulée et sa dérégulation contribue à diverses pathologies humaines. Les travaux menés au cours de ces dernières années ont révélé de nouveaux processus et mécanismes cellulaires dont la ferritinophagie, qui ont permis d'approfondir notre compréhension de l'homéostasie du fer. La ferritinophagie est une forme de macroautophagie sélective par laquelle la ferritine, la protéine de stockage du fer, est dégradée dans le lysosome. Nous présentons ici un rappel de l'homéostasie du fer puis un résumé des connaissances acquises sur la ferritinophagie et sa relation avec une nouvelle forme de mort cellulaire dépendante du fer, la ferroptose. <

\section{L'homéostasie du fer, un processus complexe hautement régulé}

Le fer est un nutriment indispensable à toute forme de vie sur terre. La capacité du fer d'alterner entre les formes oxydées (forme ferrique $\mathrm{Fe}^{3+}$, insoluble) et réduites (forme ferreux $\mathrm{Fe}^{2+}$, pro-oxydante), via la réaction de Fenton ${ }^{1}[30](\rightarrow)$, $(\rightarrow)$ Voir la Synthèse de C. Beaumont, $m / s$ $n^{\circ} 1$, janvier 2004, page 68 permet de contribuer à la formation d'espèces réactives de l'oxygène (ROS) dont la génération en excès conduit à un stress oxydatif et à l'initiation de voies de signalisation déterminantes pour la survie et la mort cellulaire. Un défaut de régulation

${ }^{1}$ La réaction de Fenton est fondée sur une oxydo-réduction du peroxyde d'hydrogène pour générer le radical $\cdot \mathrm{OH}$.

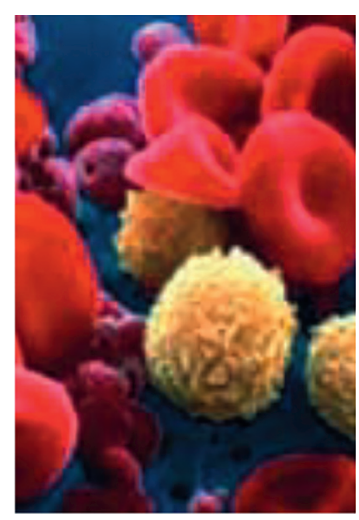

${ }^{1}$ Institut Necker-Enfants Malades (INEM), Inserm U1151-CNRS UMR 8253, 14, rue Maria Helena Vieira Da Silva, 75993 Paris Cedex 14, France;

${ }^{2}$ Université Paris DescartesSorbonne Paris Cité, F-75993 Paris, France. maryam.mehrpour@inserm.fr ahmed.hamai@inserm.fr

de l'homéostasie du fer peut donc contribuer à diverses pathologies humaines dont les maladies de surcharge en fer et le cancer. L’homéostasie du fer est donc très finement régulée à la fois au niveau systémique et au niveau de la cellule [1-5].

\section{Mécanismes de régulation de l'homéostasie systémique du fer}

L'équilibre au niveau systémique du fer est maintenu par un contrôle minutieux de son absorption intestinale et de son recyclage (sous la forme de fer héminique provenant des hématies) par les macrophages après dégradation des globules rouges sénescents. Le fer alimentaire se présente sous deux formes : le fer héminique (présent dans l'hémoglobine et la myoglobine, associé à l'hème) et le fer non héminique. L'hème est absorbé par les entérocytes duodénaux par un mécanisme qui reste encore mal connu. II est métabolisé par l'hème oxygénase 1 (HO-1) qui permet la libération de fer sous sa forme ferreux $\mathrm{Fe}^{2+}$. Pour les sources non héminique, la forme ferrique $\mathrm{Fe}^{3+}$ du fer alimentaire est réduite en fer ferreux $\mathrm{Fe}^{2+}$ dans la lumière intestinale par les réductases ferriques, comme la duodenal cytochrome b reductase (Dcytb), et transporté dans les entérocytes par le DMTl (divalent metal [ion] transporter 1), un co-transporteur de métal divalent et de protons [2, 6, 7] (Figure 1). Le fer absorbé par les entérocytes est ensuite exporté, au travers de la membrane basolatérale des cellules, dans la circulation sanguine par la ferroportine (FPN-1), le seul transporteur de fer ferreux, hautement conservé, qui est présent majoritairement dans les entérocytes, les macrophages et les hépatocytes. Ce transporteur permet donc le passage du fer du milieu intracytoplasmique vers le milieu extracellulaire (Figure 1) [8]. $\mathrm{Fe}^{2+}$, exporté par FPN-1, est ensuite réoxydé à la face basolatérale de l'entérocyte par la ferroxydase cuivredépendante, qui est liée à la membrane de la cellule, avant son chargement sur la transferrine (TF), une protéine qui circule dans le plasma et délivre le fer dans les tissus périphériques [9]. L’holotransferrine (la transferrine chargée de deux atomes de fer $\mathrm{Fe}^{3+}$ ) délivre alors le fer 


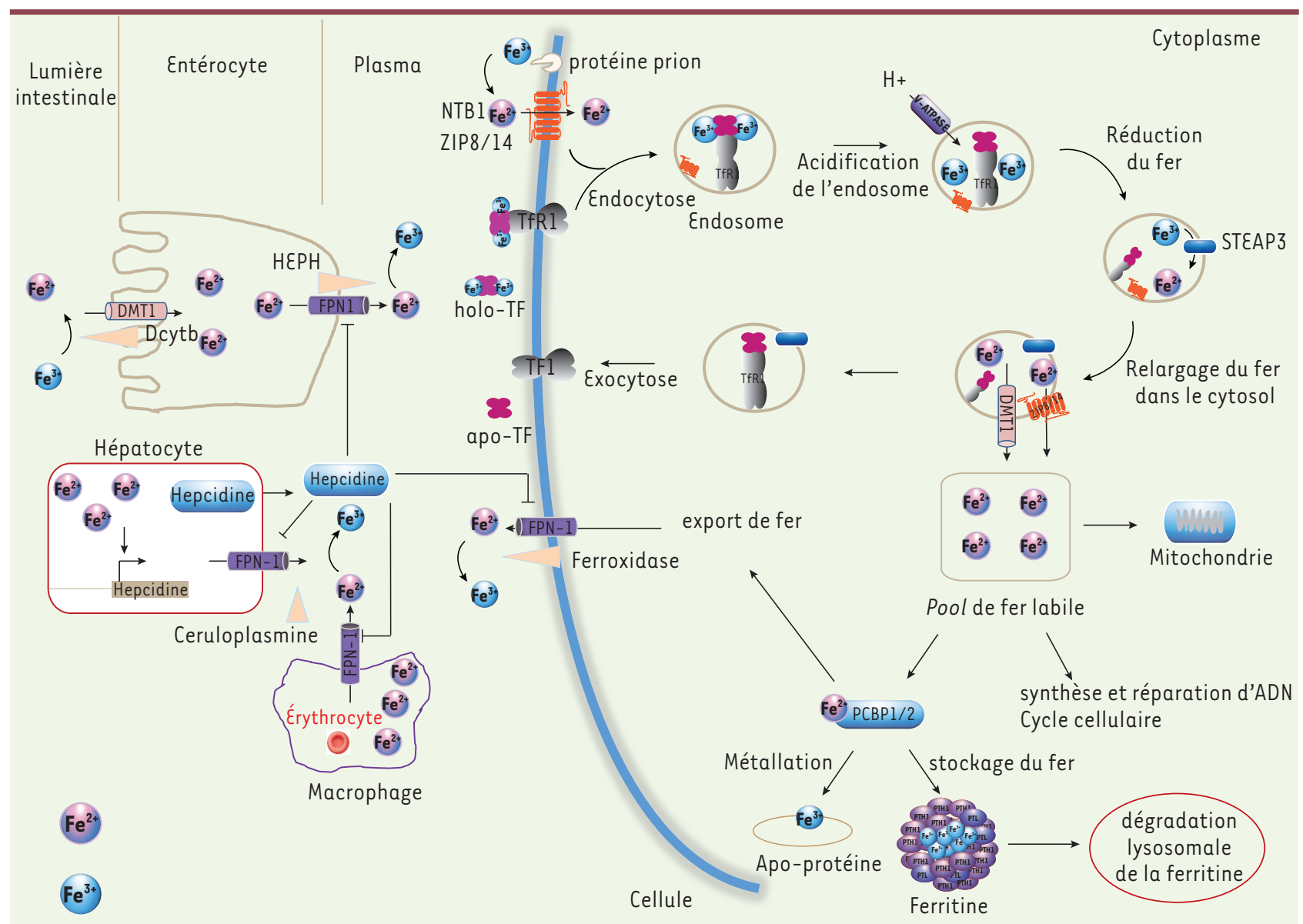

Figure 1. Schéma simplifié de l'absorption du fer et de son homéostasie. Les entérocytes absorbent le fer alimentaire grâce à l'action combinée de réductases ferriques, telles que le duodénal cytochrome b (Dcytb) et DMTl (divalent metal [ion] transporter 1)/Nramp2. Le fer ferreux repris par les entérocytes est exporté dans la circulation sanguine par la ferroportine (FPN-1). Simultanément, le Fe $\mathrm{Fe}^{2+}$ est oxydé en $\mathrm{Fe}^{3+}$ par l'héphaestine (HEPH), qui est fonctionnellement associée à la FPN-1. Dans la circulation sanguine, le Fe ${ }^{3+}$ est chargé sur l'apotransferrine (Apo-TF) pour générer la forme holotransferrine (holo-TF) associée au fer. L'holo-TF est captée par les récepteurs de la transferrine (TFRl) en fonction des besoins des différents organes et tissus. Le complexe TFRl-TF - $\left(\mathrm{Fe}^{3+}\right) 2$ est endocytosé et le fer est libéré de la TF. Fe ${ }^{3+}$ est réduit par la réductase ferrique STEAP3 (six transmembrane epithelial antigen of the prostate 3), $\mathrm{Fe}^{2+}$ est ensuite transporté vers le cytosol par DMTl pour former le pool de fer labile cytosolique (labile iron pool ou LIP). Des transporteurs ZIP8/14 (ZRT/IRTike protein 8/14) sont également impliqués dans le transport du fer par la protéine NTBI (non-transferrine bound iron) après réduction de NTBI par la protéine prion. Dans l'endosome, Fe ${ }^{2+}$ peut également être transporté vers le cytosol par ZIP8/14. Le LIP ainsi formé est utilisé pour divers besoins de la mitochondrie et de la cellule tels que la synthèse de I'ADN, de l'hème et la biogenèse des centres Fe-S. L'excès de fer est stocké par la ferritine ou exporté dans la circulation sanguine par FPN-l et une ferroxidase associée. Le fer $\mathrm{Fe}^{2+}$ du milieu intracellulaire vers le plasma est oxydé en fer $\mathrm{Fe}^{3+}$, également par la céruloplasmine plasmatique à la sortie des macrophages ou des hépatocytes. Les protéines chaperonnes PCBP1 et PCBP2 (Poly-[rC]-binding protein $1 / 2$ ) se lient au fer et le fournissent à des protéines (apo-protéines) qui nécessitent du fer non hémique comme cofacteur, comme le hypoxia-inducible factor (HIF) prolyl, les hydroxylases (métallation) à la ferritine pour le stockage, et à la DPN-1 pour son exportation. En réponse à un excès de fer circulant, I'hépatocyte synthétise l'hepcidine (HAMP), régulant ainsi négativement le fer. La HAMP induite par l'excès de fer bloque celui-ci dans différents types cellulaires comme les macrophages qui contiennent de grandes quantités de fer issues du recyclage érythrocytaire, les entérocytes, les hépatocytes, en se liant à FPN-1 entraînant son internalisation et sa dégradation. II en résulte une rétention de fer à l'intérieur des cellules et une diminution du fer plasmatique.

en se liant au récepteur TFRl (transferring receptor 1 ) qui est exprimé à la surface de la plupart des cellules. Le complexe formé (TFRl/ $\mathrm{TF}-\mathrm{Fe}^{3+}$ ) est internalisé dans un endosome dont le faible $\mathrm{pH}$ (environ $5,6)$ permet la libération des atomes de $\mathrm{Fe}^{3+}$. Le fer ferrique est alors réduit par la ferriréductase STEAP3 (six transmembrane epithelial antigen of the prostate 3) en fer ferreux puis transporté à travers la membrane endosomale par DMTl. C'est à partir du pool de fer labile et cytosolique, appelé LIP (labile iron pool) que le fer est adressé à la mitochondrie pour être incorporé dans la protoporphyrine IX et 
former l'hème, groupement prosthétique des hémoprotéines, telles que l'hémoglobine, la myoglobine, la catalase et les cytochromes, ou pour participer à l'assemblage des centres fer-soufre (nécessaires aux métalloprotéines comme les ferrédoxines, la NADH [nicotinamide adénine dinucléotide réduit] déshydrogénase, les hydrogénases, les coenzymes $\mathrm{Q}$-cytochrome $\mathrm{c}$ réductase, la xanthine oxydase, la succinate déshydrogénase et la nitrogénase). Le LIP est également utilisé par les cellules pour incorporer le fer dans le site actif de certaines enzymes (ribonucléotide réductase, prolyl hydroxylase, etc.). Le fer labile sera également capté par la ferritine afin de constituer des réserves de fer. Dans certaines cellules, il sera exporté par la FPN-1 [10]. Ce mécanisme d'export du fer vers la circulation sanguine par l'intermédiaire de la FPN-1 [11], est particulièrement actif dans les macrophages à la suite de la phagocytose des globules rouges sénescents et de la destruction de l'hème de l'hémoglobine. L'absorption intestinale du fer et son recyclage par les macrophages sont régulés négativement par l'hepcidine, l'hormone clé de l'homéostasie systémique de fer. L'expression de l'hepcidine est directement corrélée aux taux de fer intracellulaire et sérique. Une voie de signalisation complexe contrôle l'expression de l'hepcidine produite principalement par le foie. Cette hormone peptidique se lie à la FPN-1 et favorise sa phosphorylation et sa dégradation lysosomale [11]. L’hepcidine sérique régule ainsi négativement la FPN-1 dans les entérocytes duodénaux (responsables de l'absorption du fer alimentaire), les macrophages, et les hépatocytes (qui agissent comme un réservoir exportant le fer selon les besoins), conduisant à une réduction globale du fer sérique. Les niveaux de fer sont strictement régulés selon les besoins journaliers, afin de ne pas atteindre des concentrations qui seraient dangereusement élevées. Un déficit de synthèse de l'hepcidine est à l'origine de l'hémochromatose génétique et entraîne une hyperabsorption du fer au niveau de l'intestin et une surcharge tissulaire. À l'inverse, la carence en fer est la première cause d'anémie microcytaire hypochrome.

\section{Mécanismes de régulation de l’homéostasie intracellulaire du fer}

Comme au niveau systémique, l'homéostasie intracellulaire du fer est étroitement régulée au niveau post-transcriptionnel par un réseau de protéines qui dépendent du fer. Les protéines IRPI et IRP2 (iron regulatory protein), principales composantes de ce réseau, se lient sur des séquences d'ARNm, les IRE (iron responsive element) qui sont des structures tige-boucle situées dans des régions 5' ou 3' non traduites (UTR) des ARNm. Les IRP ont pour rôle d'adapter, selon la charge en fer, l'expression des protéines qui régulent l'importation (TFRl, DMTl), le stockage (ferritine et ses deux sous-unités FTHl [ferritin heavy chain 1] et FTL [ferritin light chain])2, et l'exportation (FPN-1) du fer présent dans la cellule [12]. Selon qu'il est situé à l'extrémité 5' UTR ou 3' UTR de l'ARNm, l'interaction entre l'élément IRE et la protéine IRP engendrera des effets opposés sur l'expression du gène cible. La

${ }^{2}$ La ferritine est une molécule multimérique disposant de 24 sous-unités lourdes (FTH) et légères (FTL) et capable de chélater 4000-4500 atomes de fer par molécule. liaison d'une IRP à un IRE en 5' limite la traduction de I'ARNm et donc diminue l'expression du facteur correspondant. En revanche, la fixation d'une IRP à un IRE en 3' stabilise l'ARNm en le protégeant des ribonucléases intracellulaires augmentant son expression. Dans des conditions de carence en fer, les IRP se lient sur les éléments IRE situés en 5 ' des ARNm de la ferritine et de la FPN-1 pour réprimer leur traduction, et sur les éléments IRE situés en 3' de l'ARNm de TFRl pour limiter sa dégradation. En conditions d'excès de fer, un complexe fer/soufre se forme au niveau de l'IRPl entraînant un changement conformationnel qui l'empêche de se lier à un IRE, ce qui entraîne une augmentation de la synthèse de la ferritine et de FPN-l et une dégradation accrue de I'ARNm de TFRl. En revanche, dans ces conditions, IRP2 est polyubiquitinylée et détruite par le protéasome. En contrôlant l'importation, le stockage et l'exportation de fer, les protéines IRP veillent à ce que les besoins métaboliques en fer soient respectés, tout en minimisant les effets toxiques induits par un excès en fer.

Des travaux récents ont mis en évidence la possibilité pour le fer ferreux $\mathrm{Fe}^{2+}$ (et d'autres ions métalliques) de se lier directement à la tige-boucle IRE, en 5' UTR, et d'induire un changement conformationnel de la structure qui entraîne une diminution de l'affinité de I'élément pour les IRP et une augmentation de liaison du complexe d'initiation de la traduction, elF4F (eukaryotic initiation factor 4F) [4]. La structure de I'IRE contribue donc en elle-même à la régulation posttranscriptionnelle de l'expression génique du réseau de protéines dépendantes du fer.

\section{Nouvelles protéines impliquées dans l'absorption et le trafic du fer}

Au cours de ces dernières années, plusieurs protéines dont des transporteurs de fer et des molécules chaperonnes ont été identifiées comme de nouveaux acteurs clés du trafic intracellulaire du fer [4].

L'importance de transporteurs de la famille ZRT/IRTlike protein (ZIP), ZIP8 et ZIP14, a ainsi été montrée pour le transport du fer non lié à la transférine (ou NTBI pour non-transferrine bound iron) après la réduction de ce dernier par la protéine prion [4]. Le NTBI est une forme particulière du fer plasmatique présente dans des situations de forte surcharge en fer, en particulier après transfusion. L'expression de ZIP8, et de ZIP14, est régulée différemment de celle des autres gènes codant des transporteurs de fer, tels que TFRl et DMTl, tous les deux régulés au niveau post-transcriptionnel. La stabilité des ARNm de ces protéines n'est pas affectée par le fer mais le niveau d'expression des protéines ZIP est 
A

Condition basale ou en carence en fer ou lors de la stimulation de la ferritinophagie
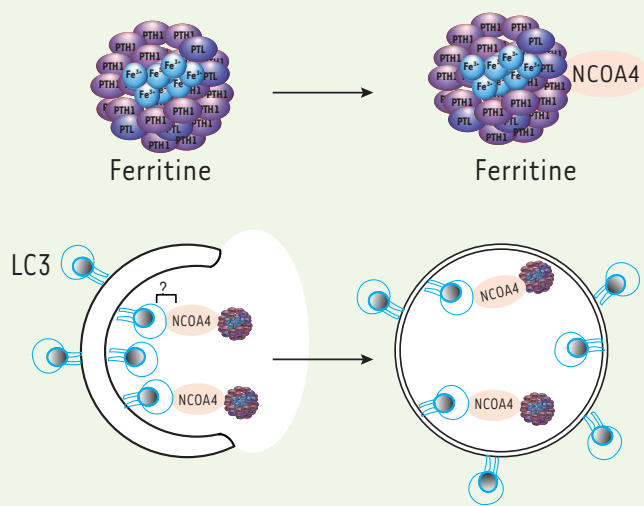

Phagophore

\section{Condition riche en fer}

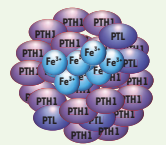

Ferritine stabilisée

(e3) NCOA4

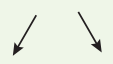

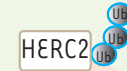

? (6.) NCOA4

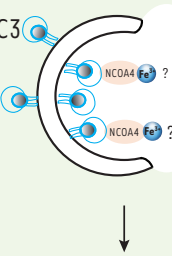

Dégradation de NCOA4 par la macroautophagie

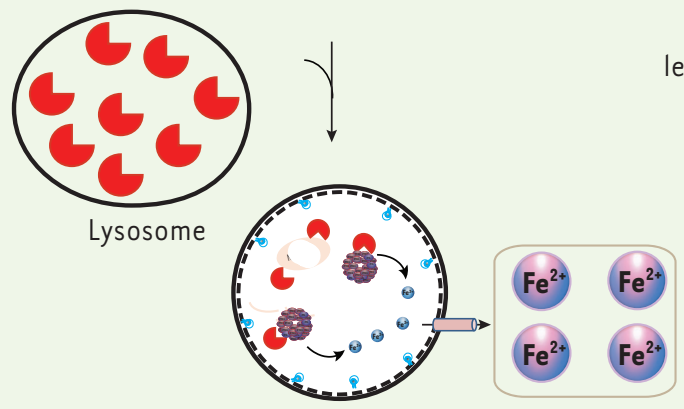

Dégradation de NCOA4 par le système ubiquitine protéasome

Pool de fer labile

Diminution de NCOA4

et de la ferritinophagie

Figure 2. Schéma simplifié de la ferritinophagie dépendante de NCOA4. A. En condition basale ou en carence de fer, le fer Fe $e^{3+}$ est stocké dans la ferritine, une molécule multimérique capable de chélater 4000-4500 atomes de fer par molécule. NCOA4 (coactivateur de récepteur nucléaire 4) reconnaît la ferritine et l'achemine au phagophore. La nature moléculaire de l'interaction NCOA4-autophagosome est incertaine, mais probablement implique une interaction avec LC3 (microtubule-associated protein 1A/1B-light chain 3)-II/GABARAPL (gamma-aminobutyric acid receptorassociated protein) $1 / 2$ potentiellement via un motif LIR (LC3-interacting region) d'expression non-canonique. La dégradation de la ferritine dans l'autolysosome libère le fer $\mathrm{Fe}^{2+}$, qui est exporté vers le cytosol. B. Le niveau de NCOA4 (nuclear receptor coactivator 4) ainsi que la ferritinophagie sont régulés par la quantité du fer dans la cellule. En conditions riches en fer, NCOA4 est reconnu par HERC2 (HECT and RLD domain-containing $\varepsilon 3$ ubiquitin protein ligase 2), une ubiquitine ligase $\varepsilon 3$, d'une manière dépendante du fer, et ciblé pour sa dégradation par le protéasome. En parallèle, NCOA4 se lie au fer (la forme ferreux ou ferrique n'est pas encore déterminée) et emprunte la voie de macroautophagie non sélective pour être dégradé. Le niveau plus faible de NCOA4 dans la cellule entraine une diminution de la ferritinophagie.

positivement corrélé au statut en fer. En effet, la carence en fer diminue la quantité de protéines ZIP8 et ZIP14 tandis que la surcharge en fer l'augmente. Le rôle de ces nouvelles protéines dans des contextes comme les états pathologiques avec dérégulation du métabolisme du fer (anémie, maladies de surcharge en fer, cancer, maladies neurodégénératives, etc.) reste à explorer.

Les protéines chaperonnes PCBP1 et PCBP2 (Poly-[rC]-binding protein 1/2) sont des protéines adaptatrices multifonctionnelles qui lient le fer et le transmettent à la ferritine à des fins de réserve. PCBPl et PCBP2 peuvent également fournir le fer à d'autres protéines qui nécessitent du fer non hémique comme cofacteur comme l'hypoxia-inducible factor (HIF) prolyl, les asparagyle hydroxylases à métallate et l'hydroxylase désoxyhypusine. PCBP2 peut se lier à DMTl, transporteur de fer. Le transfert de fer de DMT1 à PCBP2 est réalisé par l'interaction du second domaine $\mathrm{KH}$ (K-homologous) de $\mathrm{PCBP}^{3}$ avec le domaine cytoplasmique $\mathrm{N}$-terminal de DMTl [13]. Après le transfert de $\mathrm{Fe}^{2+}, \mathrm{PCBP} 2$ se dissocie de DMTl (Figure 1). Contrairement à PCBP2, PCPB1 n'interagit pas avec DMTl et le mécanisme d'acquisition du fer par l'apo-PCBPI (dépourvue de fer) est encore indéterminé.

${ }^{3}$ PCBP2 contient 3 domaines $K$-homologous $(\mathrm{KH})$ possiblement impliqués dans la liaison à l'ARN. 
De nombreuses questions concernant les rôles et les mécanismes des protéines PCBP dans l'homéostasie du fer restent encore sans réponse. II a été noté, en particulier, que PCBP2 peut se lier à FPN-1, l'exportateur de fer, mais la caractérisation de cette association reste incomplète et les effets que PCBP2 peut induire sur FPN-1 pour l'exportation de fer restent à définir. $\varepsilon$ n tout état de cause, la description récente de ces protéines chaperonnes et de leur rôle dans l'homéostasie du fer pourrait remettre en question l'existence du LIP, le pool de fer labile et cytosolique.

\section{L'adressage de la ferritine pour sa dégradation dans les lysosomes dépend, ou non, des voies de signalisation de la macroautophagie}

La macroautophagie est la voie principale de dégradation dans le lysosome de constituants cytosoliques (macromolécules, agrégats protéiques et lipidiques) et d'organites (en excès ou endommagés). Ce processus catabolique, sous le contrôle hiérarchisé des gènes de la famille ATG (autophagy-related gene), est initié par la formation d'une structure à double membrane dans le cytosol, appelée phagophore. Cette structure s'allonge, séquestre les différents éléments, puis se referme pour former une vésicule à double membrane appelée autophagosome qui fusionne avec le lysosome pour donner l'autolysosome. Après fusion, le substrat est dégradé par les hydrolases lysosomales et les produits de dégradation sont recyclés pour la synthèse de nouvelles macromolécules et la production de l'énergie, ou mis en réserve. Pendant le processus d'allongement du phagophore et la fermeture de l'autophagosome, la phosphoinositide 3-kinase de classe III Vps34 phosphoryle le phosphatidylinositol ( $\mathrm{PI}$ ) pour générer le phosphatidylinositol 3-phosphate (PI3P). La production de PI3P représente le signal d'initiation de la formation de l'autophagosome. Quatre membres de la famille des protéines WIPI (WD repeat domain phosphoinositide-interacting protein) humaines (WIPI1-4) sont indispensables pour décoder le signal que représente le PI3P au niveau de I'autophagosome naissant. Les protéines WIPI sont recrutées au niveau du site de formation du phagophore où elles se lient spécifiquement à PI3P. WIPI2B favorise ainsi la conjugaison (encore appelée lipidation) du LC3 (microtubule-associated protein 1A/IB-light chain 3) ou de GABARAP (gamma-aminobutyric acid receptor-associated protein) à la phosphatidyléthanolamine (PE) par le recrutement du complexe ATG12-5/ATG16 permettant l'allongement du phagophore [14]. Si le rôle de WIPIl et 2 dans la biogenèse d'autophagosome est bien étudié, celui de WIPI3 et 4 reste à déterminer dans ce processus.

Le premier lien, indirect, établi entre l'homéostasie du fer et la macroautophagie, chez l'homme, repose sur des études récentes. Elles relient en effet un défaut de renouvellement de la ferritine avec un trouble neurodégénératif, l'encéphalopathie statique de l'enfance avec neuro-dégénérescence chez l'adulte (static encephalopathy of childhood with neurodegeneration in adulthood, SENDA). Cette maladie se caractérise par l'accumulation de dépôts de fer dans les noyaux gris centraux, avec pour origine des mutations au niveau du gène WDR45 (WD repeat domain 45, autre nom de WIPI4). Ces résultats reliant accumulation de fer et implication de gènes de la famille WIPI, soulignent donc l'importance de l'autophagie dans l'homéostasie du fer et sa biodisponibilité $[15,16]$. Comme nous l'avons vu, la régulation du taux de fer dans les cellules est maintenue par un réseau de protéines dépendant du fer. L'excès de fer est stocké dans la ferritine. Lors d'une réduction des apports, sa biodisponibilité est maintenue par la dégradation de la ferritine dans le lysosome et sa libération. La ferritine sert donc de réservoir de fer pour la cellule. Elle est aussi à la base d'un mécanisme de prévention de la génération de radicaux libres via la réaction de Fenton.

\section{Macroautophagie et ferritine}

Le rôle direct de la macroautophagie dans la dégradation de la ferritine lors de la déplétion en fer a été initialement mis en évidence par des études utilisant des fibroblastes de souris invalidés pour le gène Atg5 qui code l'une des protéines ATG [17]. Par la suite, des études ultrastructurales réalisées sur des fibroblastes de souris et des cellules humaines de la lignée Hela, invalidées pour les gènes d'autophagie comme ATG9 ou FIP200 (FAK family kinase-interacting protein of 200 $k D a$ ) ont permis de montrer que des amas de ferritine étaient accumulés au niveau du site de formation de l'autophagosome, colocalisés avec la protéine p62, un substrat de la macroautophagie, montrant que la ferritine peut être recrutée sur le site de formation de l'autophagosome indépendamment des protéines ATG [18].

Le mécanisme par lequel la ferritine est ciblée vers la dégradation autophagique a été élucidé par deux études de protéomique $[19,20]$ qui ont permis d'identifier le coactivateur de récepteur nucléaire 4 (NCOA4, nuclear receptor coactivator 4) comme le récepteur spécifique de la ferritine qui l'adresse au lysosome pour sa dégradation. Ce processus, appelé «ferritinophagie », est important pour le maintien de l'homéostasie cellulaire du fer. En effet, l'invalidation du gène Ncoa4 entraîne une augmentation d'expression d'IRP2 et de $T F R 1$, probablement par diminution du fer labile suite à l'accumulation de ferritine. Les souris dont le gène Ncoa4 est invalidé présentent également une accumulation importante de fer dans les macrophages de rate indiquant une altération du stockage et du recyclage du fer [19]. La dégradation de la ferritine par ferritinophagie dépendante de NCOA4 est donc essentielle pour I'homéostasie du fer dans la rate [20].

La fonction, nouvellement identifiée, de NCOA4 comme récepteur de la ferritinophagie est remarquablement différente de ses rôles, identifiés à l'origine, comme coactivateur de récepteurs des androgènes [21]. NCOA4 
est en fait un substrat de la macroautophagie lorsque cette dernière est inhibée par un inhibiteur hautement sélectif du complexe Vps34, initiateur du processus d'autophagie [20]. NCOA4 interagit avec l'ensemble du complexe ferritine via la chaîne lourde de la protéine.

La découverte de récepteurs spécifiques de l'autophagie (ou des protéines adaptatrices autophagiques), qui séquestrent le matériel à dégrader (encore appelé substrat) dans le phagophore, a été décisive pour la compréhension des mécanismes de la macroautophagie sélective, dont la ferritinophagie. L'ubiquitination est souvent une condition préalable à la reconnaissance du substrat. Elle détermine également la sélectivité de la macroautophagie chez les eucaryotes supérieurs. Différents types de récepteurs ont été décrits en fonction du type d'autophagie sélective. Deux catégories se dégagent néanmoins en fonction de la façon dont ils reconnaissent le substrat, dépendant ou indépendant de l'ubiquitine. Cette sélectivité repose également sur le motif de zone d'interaction LIR (LC3-interacting region) qui présente la séquence typique $[\mathrm{W} / \mathrm{F} / \mathrm{Y}] \mathrm{xx}[\mathrm{L} / \mathrm{I} / \mathrm{V}]$. Ce motif assure le ciblage des récepteurs de l'autophagie vers LC3 qui est ancré dans la membrane du phagophore. Les protéines qui présentent la région LIR comprennent les récepteurs d'autophagie, certaines protéines ATG, les protéines associées aux vésicules et à leur transport, les GAP (Rab GTPase-activating protein) et des protéines de signalisation qui sont dégradées par autophagie sélective [22]. Bien que NCOA4 ne possède pas de domaine LIR, sa déplétion, par ARN interférence, prévient l'acheminement et la dégradation lysosomale de la ferritine par ferritinophagie l'impliquant, de ce fait, comme un récepteur participant au renouvellement de la ferritine (Figure 2). L'absence de domaine ubiquitin-binding domain (UBD) sur NCOA4 suggère que la dégradation de la ferritine par la ferritinophagie dépendante de NOCA4 est une forme de macroautophagie sélective qui n'implique pas l'ubiquitine [23]. Des études ultérieures ont montré que NCOA4 interagit directement avec FTHl via son domaine C-terminal (1489/W497) et le résidu arginine 23 de $\mathrm{FTHl}$, et des expériences de mutagenèse dirigée ont mis en évidence la nécessaire implication de cette région dans la ferritinophagie. Dans la cellule, la quantité de NCOA4 est sous le double contrôle de l'autophagie et du système ubiquitine-protéasome et son renouvellement, qui dépend de l'ubiquitine et du fer, est déclenché dans des conditions riches en fer et implique une interaction avec la ligase ubiquitine HERC2 (HECT and RLD domain-containing $\varepsilon 3$ ubiquitin protein ligase 2). La ligase ubiquitine HERC2, qui a été identifiée par des études de protéomique, reconnaît NCOA4 par son domaine CUL7 (Cullin 7)-homology, avec pour résultat, la réduction du niveau de NCOA4, ce qui augmente la ferritine disponible pour la capture du fer. Étonnamment, le domaine C-terminal de NCOA4 (1489/W497) lie le fer, et l'état de NCOA4, associé ou non au fer, détermine sa liaison avec HERC2, ce qui suggère l'existence d'un commutateur à l'origine du renouvellement de NCOA4 qui dépend du fer.

\section{Ferritinophagie et érythropoïèse}

Des études utilisant le modèle du poisson zèbre et la lignée cellulaire érythroleucémique et hémine inductible (les cellules K562), ont montré que la ferritinophagie dépendante de NCOA4 est impor- tante pour l'érythropoïèse in vivo et in vitro. Les liens initiaux entre NCOA4 et l'érythropoïèse reposaient sur une étude montrant une expression plus élevée d'ARNm de NCOA4 aux sites de l'érythropoïèse, au cours du développement du poisson zèbre. Lors de la différenciation érythroïde, la majorité du fer obtenu par endocytose de l'holo-transferrine pourrait donc être mise en réserve par la ferritine avant sa mobilisation via la ferritinophagie dépendante de NCOA4, pour la synthèse de l'hème [23]. La ferritinophagie dépendante de NCOA4, apparaît ainsi essentielle dans la régulation du métabolisme du fer cellulaire et dans l'organisme.

\section{Ferritinophagie et stress oxydatif}

Le rôle de NCOA4 dans la régulation de I'homéostasie systémique de fer a été examiné dans un modèle de souris transgénique déficiente pour NCOA4 [24]. Ces souris, comme attendu, accumulent de façon inappropriée la ferritine chargée en fer dans tous les tissus évalués. Ne pouvant mobiliser le fer à partir de la ferritine, les souris développent une anémie hypochrome microcytaire qui est considérablement aggravée quand elles sont nourries avec un régime alimentaire pauvre en fer. Nourries avec un régime riche en fer, elles présentent une augmentation significative des dépôts de ferritine, des concentrations sériques élevées d'aspartate aminotransférase et d'alanine aminotransférase hépatique, un accroissement de la dégénérescence graisseuse du foie, et une mort prématurée, par rapport aux souris sauvages. L'hypothèse soutenue par les auteurs de ces études est que l'augmentation du niveau de base en fer dans les tissus des souris déficientes en NCOA4 sature des voies antioxydantes, ce qui entraîne les dommages hépatiques observés. Les protéines impliquées dans la réponse antioxydante comme la superoxyde dismutase et la glutathion peroxydase sont en effet exprimées à des niveaux significativement plus élevés chez les souris n'exprimant pas NCOA4. La ferritinophagie peut donc également jouer un rôle important dans la modulation du stress oxydatif au niveau de l'organisme. Il reste cependant à déterminer l'importance physiologique de la ferritinophagie dépendante de NCOA4 dans d'autres types de tissus, en particulier lors du développement du système nerveux central et au cours des maladies neurodégénératives.

Deux études mettent en évidence une dégradation possible de la ferritine en présence d'inhibiteur de la kinase PI3K classe III (la 3-méthyladénine), indiquant que sa dégradation peut être indépendante de la macroautophagie canonique $[17,25]$. Ces résultats laissent ainsi 
penser, qu'à côté de la ferritinophagie, d'autres voies de dégradations lysosomales existent pour la ferritine, de même que la voie du protéasome induite par certains chélateurs de fer ou la surexpression de la ferroportine $[25,26]$.

\section{Le fer, un médiateur important de la mort cellulaire}

L'effet toxique principal du fer repose sur sa capacité à induire la formation de radicaux libres, lors de la réaction de Fenton avec, pour conséquence, une peroxydation lipidique. Récemment, une nouvelle forme de mort cellulaire a été décrite : la ferroptose. La ferroptose est une voie de mort cellulaire initialement décrite comme une voie non-apoptotique, non-nécrotique et non-autophagique [27]. La ferroptose semble se produire à la suite de deux processus touchant la cellule : la perturbation du pouvoir antioxydant cellulaire, et l'augmentation de la quantité de fer intracellulaire. Il en résulte une peroxydation des lipides de la membrane plasmique. La formation des ROS est induite par le fer et par les NADPH (nicotinamide adénine dinucléotide phosphate réduit) oxydases, des enzymes de la famille Nox contenant un hème. Elle conduit in fine à une accumulation de peroxydes lipidiques toxiques. Les composés chimiques inducteurs de la ferroptose, comme l'érastine et le sorafenib, de même que la signalisation de $p 53$, perturbent le transport de la cystéine, ce qui produit un appauvrissement de glutathion cellulaire antioxydant (GSH, forme réduite). Cet épuisement de GSH se produit également au cours de lésions d'ischémie/reperfusion, processus au cours duquel le stress oxydatif submerge la capacité antioxydante de la cellule. La perte de GSH inhibe la glutathion peroxydase 4 (GPX4), une enzyme clé de la régulation de la ferroptose. GPX4 empêche la suractivation de lipooxygénase et la peroxydation lipidique membranaire et ainsi protège la cellule de la mort cellulaire. En utilisant deux molécules de GSH, GPX4 réduit les hydroperoxyde phospholipides $(\mathrm{L}-\mathrm{OOH})$ en hydroxyde phospholipides $(\mathrm{L}-\mathrm{OH})$. GPX4 limite ainsi à partir de $\mathrm{L}-\mathrm{OOH}$ la formation de radicaux alkoxy lipidiques ( $L-0 \cdot$ ) hautement réactifs et dépendante du fer. À ce jour, c'est le seul système cellulaire décrit responsable de la réparation efficace des phospholipides oxydés. La ferroptose peut être inhibée par la chélation du fer, ainsi que par plusieurs petites molécules (comme la ferrostatin-1, la liproxstatin-1, etc.). En revanche, les inhibiteurs de l'apoptose (en particulier Z-VAD-FMK, un inhibiteur des caspases) ou de la nécroptose (la nécrostatine-1) n'ont pas d'effet sur la ferroptose. Bien que le rôle exact du fer ne soit pas entièrement élucidé, il existe un faisceau d'arguments qui montre l'implication du fer dans cette forme de mort cellulaire. Ainsi, l'augmentation du fer intracellulaire par un traitement par l'érastine et au cours de lésions d'ischémie-reperfusion contribuent à l'accumulation des hydroperoxydes lipidiques et à la mort par ferroptose. IRP2 a été également identifié comme un gène essentiel pour l'induction de la ferroptose dans les cellules de la lignée humaine de fibrosarcome HT-1080, probablement en raison de son rôle dans l'accumulation de fer et la régulation des gènes du métabolisme du fer, par son interaction avec les IRE. Les inhibiteurs de la ferroptose agissent soit en empêchant la dégradation oxidative des lipides (avec la ferrostatine 1), soit en diminuant le fer intracellulaire (avec des chélateurs de fer, ou la protéine de choc thermique Bl ([HSPBl]).
Les études portant sur la relation entre ferritinophagie et ferroptose n'en sont qu'à leur début. II a cependant été montré qu'en dégradant la ferritine, dans des fibroblastes et des cellules cancéreuses, la ferritinophagie contribue à la ferroptose. De même, I'invalidation des gènes d'autophagie comme ATG5 ou ATG7 limite la ferroptose induite par l'érastine et la déplétion de NCOA4 prévient la dégradation de la ferritine et la ferroptose. À l'opposé, la surexpression du gène NCOA4 entraîne une augmentation de la dégradation de la ferritine et favorise la ferroptose [28]. Une étude utilisant des fibroblastes en culture et l'invalidation du gène Atgl3 a confirmé l'implication de la ferritinophagie dans la ferroptose [29]. Ces résultats fournissent ainsi un nouvel aperçu de I'interaction entre macroautophagie et mort cellulaire programmée.

\section{Conclusions}

Au cours de ces dernières années, les connaissances sur la régulation du fer dans des conditions physiologiques et pathologiques (cancers et maladies neurodégénératives) ont été approfondies et élargies. De nouveaux mécanismes impliquant le fer, comme la ferritinophagie et la ferroptose, ont été identifiés et l'exploitation de ces connaissances à des fins thérapeutiques a débuté. D'autres travaux permettant de comprendre l'importance de la ferritinophagie in vivo devraient conduire à l'identification des compartiments tissulaires impliqués dans ce processus et à élucider les mécanismes de dérégulation de la ferritinophagie à l'origine de maladies. $\diamond$

\section{SUMMARY}

\section{Autophagy and iron homeostasis}

Iron is an essential nutrient to life. However, the ability of iron to cycle between the oxidized and reduced forms contributes to the formation of reactive oxygen species. The generation of free radicals leads to oxidative stress and the initiation of signaling pathways involved in cell survival or cell death. The iron homeostasis is very carefully regulated and dysregulation of iron metabolism contributes to various human pathologies. The work carried out in recent years has revealed new cellular processes and mechanisms like ferritinophagy, that deepen our understanding of iron homeostasis. Ferritinophagy is a form of selective macroautophagy whereby ferritin, an iron storage protein, is degraded in the lysosome. Here, we describe iron homeostasis and review recent discoveries regarding the mechanism of ferritinophagy and its relationship to a new form of cell-death iron-dependent, the ferroptosis. $\diamond$ 
